\title{
Texture Based Quality Analysis of Simulated Synthetic Ultrasound Images Using Local Binary Patterns ${ }^{\dagger}$
}

\author{
Prerna Singh ${ }^{1, *}$ (1), Ramakrishnan Mukundan ${ }^{1}$ and Rex De Ryke ${ }^{2}$ \\ 1 Department of Computer Science and Software Engineering, University of Canterbury, \\ Christchurch 8041, New Zealand; mukundan@canterbury.ac.nz \\ 2 Radiology Services, Canterbury District Health Board, Christchurch 8140, New Zealand; \\ Rex.DeRyke@cdhb.health.nz \\ * Correspondence: prerna.singh@pg.canterbury.ac.nz; Tel.: +64-2041175855 \\ + This paper is an extended version of our paper published in (Speckle Simulation and Quality Evaluation of \\ Synthetic Ultrasound Images. In Communications in Computer and Information Science, Processing of the \\ Medical Image Understanding and Analysis. (MIUA), Edinburgh, UK, 11-13 July 2017; Valdés Hernández, M., \\ González-Castro, V., Eds.; Springer: Cham, Switzerland, 2017; Volume 723, pp. 74-85.
}

Received: 28 October 2017; Accepted: 18 December 2017; Published: 21 December 2017

\begin{abstract}
Speckle noise reduction is an important area of research in the field of ultrasound image processing. Several algorithms for speckle noise characterization and analysis have been recently proposed in the area. Synthetic ultrasound images can play a key role in noise evaluation methods as they can be used to generate a variety of speckle noise models under different interpolation and sampling schemes, and can also provide valuable ground truth data for estimating the accuracy of the chosen methods. However, not much work has been done in the area of modeling synthetic ultrasound images, and in simulating speckle noise generation to get images that are as close as possible to real ultrasound images. An important aspect of simulated synthetic ultrasound images is the requirement for extensive quality assessment for ensuring that they have the texture characteristics and gray-tone features of real images. This paper presents texture feature analysis of synthetic ultrasound images using local binary patterns (LBP) and demonstrates the usefulness of a set of LBP features for image quality assessment. Experimental results presented in the paper clearly show how these features could provide an accurate quality metric that correlates very well with subjective evaluations performed by clinical experts.
\end{abstract}

Keywords: ultrasound image analysis; speckle noise; synthetic ultrasound images; texture features; local binary patterns; image quality assessment

\section{Introduction}

Ultrasound images are known to have poor signal-to-noise ratio, yet they are low cost non-invasive techniques in diagnostic radiology and hence extensively used in clinical applications. Several new ultrasound image analysis algorithms are currently being researched for noise reduction [1-3], segmentation [4], registration and volume reconstruction [5]. Online ultrasound image databases are now becoming increasingly available and this has greatly benefitted researchers in obtaining reference images for testing and evaluating algorithms [5-7].

The speckle noise in ultrasound images degrades the fine details and edge definitions, and limits the contrast resolution by making it difficult to detect small and low contrast lesions in the body. Therefore, algorithms for ultrasound image filtering and analysis primarily focus on the characteristics of speckle noise and try to minimize its effects on image interpretation [8]. To analyse the effectiveness or accuracy of speckle reduction techniques, it is necessary to add controlled noise to ideal noiseless images [2]. In the absence of such noiseless ground truth images, the evaluation of despeckling 
algorithms is usually done by generating highly artificial gray tone patterns (stripes, rings etc.), or by using standard image sets (Lena, Mandrill etc.) that bear no resemblance with ultrasound image characteristics. This paper addresses the need for generating accurate synthetic models of ultrasound image formation for applications in speckle noise analysis. A synthetic ultrasound image can be sampled using a configuration of points that correspond to either linear or sector scan modes of ultrasound imaging, and interpolated later after generating speckle noise at the sampled points to obtain visually realistic effects. Synthetic images can therefore be used to generate simulated ultrasound images with a wide range of image and noise characteristics useful for filtering methods and noise analysis.

Statistical and empirical methods of generating speckle lack realism since several important characteristics of the image acquisition models are often ignored. There are only very limited algorithms reported in literature for speckle simulation based on image acquisition modeling. Perreault and Auclair-Fortier [9] proposed an efficient simulation model of ultrasound images based on a radial-polar configuration of sampling points and a speckle noise simulation algorithm. We extend their work by considering different types of sampling and interpolation schemes and by performing detailed experimental analysis to compare their effectiveness in producing realistic speckle simulation. Their work used images of Lena and Barbara as the basis for generating sectoral images with speckle noise to demonstrate the outputs of their algorithm. However, for generating highly realistic synthetic models, we require images that have texture features and intensity characteristics that closely match the features of real ultrasound images. We would like to emphasize here that the goal of the research work presented in the paper is not to develop an image synthesis system for generating images that could be used in place of real ultrasound images. The proposed system is designed as a versatile framework allowing several parameter variations that could be beneficial for researchers in generating images with texture and noise characteristics closely matching the features of real ultrasound images. Therefore, a very important aspect of synthetic image modeling algorithms is quality assessment. In the proposed method, the base synthetic image is modified as outlined above using the acquisition model, speckle noise simulation and interpolation of the sampled points. Second order texture feature analysis using Gray level co-occurrence matrix (GLCM) has been already performed in our recently proposed work [10], where we felt the need for more robust texture descriptors that would show consistent behavior with variation of image quality induced by changes in the modeling parameters. This paper addresses this problem by considering local binary patterns (LBP) for representing the texture content in the images. To the authors' knowledge, no prior work has been reported on image texture feature based quality assessment of realistic synthetic ultrasound images using local binary patterns. In this paper, the quality of the generated synthetic image texture features are analyzed using LBP [11,12], and correlated with subjective evaluation scores assigned by clinicians. Our paper address this main gap in the area of performance evaluation of denoising algorithms where methods for generating ground truth data having the important texture and noise characteristics of real ultrasound images are not currently available. This proposed framework also allows noise to be added in a controlled way which is essential for evaluation using quality metrics.

The paper presents the complete framework for the development of synthetic ultrasound images including the set of processes in both simulation and evaluation stages. It also presents the results of an exhaustive experimental analysis of texture features using LBP for each of the three sampling methods. The presented results clearly show the usefulness of LBP in accurately characterizing the texture features and therefore the overall quality of modelled synthetic images. This paper is organized as follows: The next section gives a brief outline of the images used and the methods in the processing pipeline. Section 3 describes the simulation model in detail. Section 4 presents the synthetic ultrasound images with variations of modeling parameters. Section 5 gives an overview of local binary patterns. Section 6 presents experimental results and their evaluations using LBP. Section 7 gives a summary of the work presented in the paper and outlines future directions. 


\section{Materials and Methods}

For experimental work presented in the paper, the reference images of real ultrasound images were sourced from the online ultrasound image gallery [7]. These are ultrasound scans of the liver, and have very similar image features, intensity distribution and noise content. Figure 1 shows three such reference images. The texture features extracted from these images will be compared in later sections with those of the generated synthetic images.

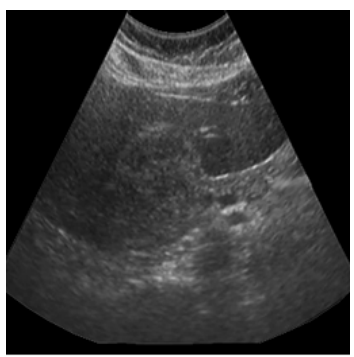

Liver 1

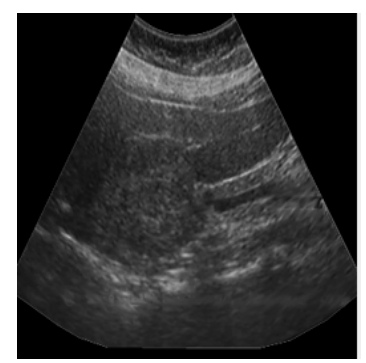

Liver 2

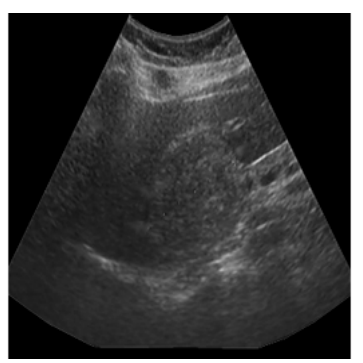

Liver 3

Figure 1. Reference ultrasound images [7] used in our work.

The important visual features of the three reference images in Figure 1 were considered by an artist to render a base image as shown in Figure 2. This image is used as the input to the simulation pipeline shown in Figure 3.

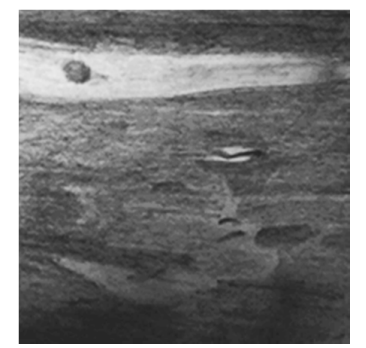

Artist rendered image

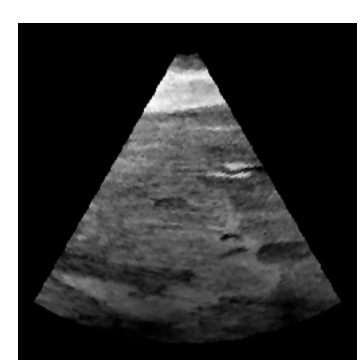

Sector Image

Figure 2. Artist rendered synthetic image and its cropped version using a sector region.

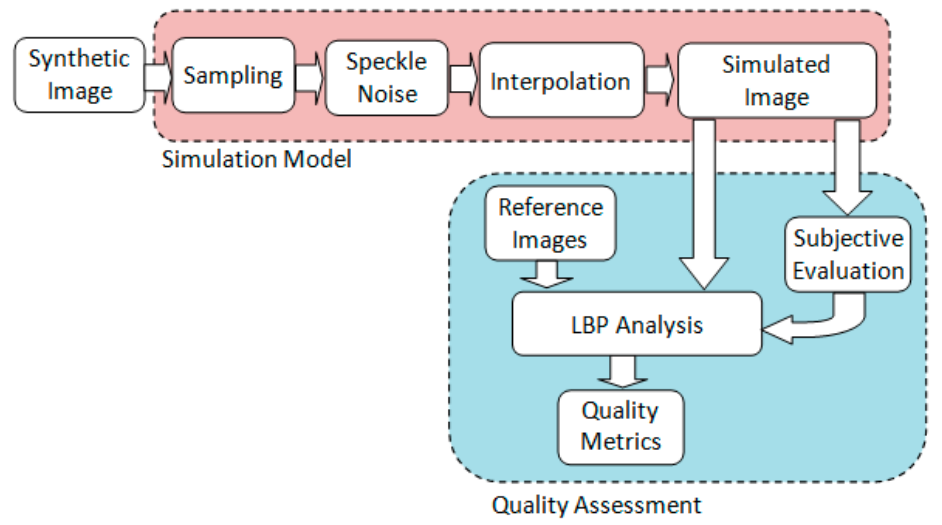

Figure 3. The simulation and evaluation stages of the processing pipeline.

The main methods used in the speckle simulation modeling and evaluation pipeline are depicted in Figure 3. Within the simulation model, the synthetic image is first sampled based on an acquisition model, speckle noise is then generated at the sampled points, and an interpolation algorithm used to fill the sector scan region. The evaluation model uses image quality metrics computed for the 
output are then compared with those of the reference ultrasound images for a quantitative assessment of the quality of the final synthetic images. A subjective evaluation is also performed using expert sonographers. In consideration of subjective evaluation, texture feature analysis is performed using local binary patterns in order to validate subjective assessment and equate results to human perception of texture.

The processing stages within the simulation model are further elaborated in the next section.

\section{The Modeling and Speckle Simulation}

The first stage of the sampling model is the method that generates a set of points at a coarse spatial resolution. The configuration of points models the loss of resolution of the ultrasound image due to pulse length, and also the scanning mode (sector or linear). One of the original contributions in this field is the paper by Perreault and Auclair-Fortier [9], where a radial-polar sampling model was introduced. We extend their work and propose three types of sampling methods called radial-polar, radial-uniform, and uniform grid. The first two are closely related to sector scan, while the third corresponds to a sampling in linear orthogonal directions (Figure 4).

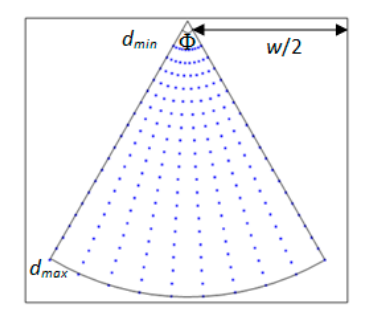

Radial-Polar

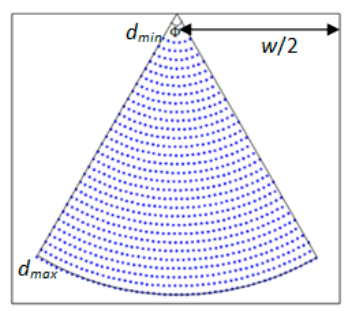

Radial-Uniform

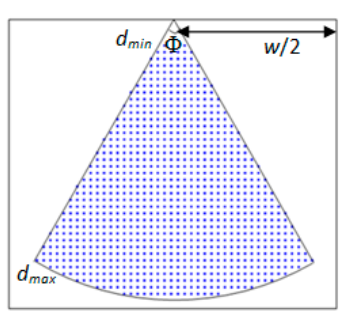

Uniform Grid

Figure 4. Sampling models that can be used in simulating speckle noise ([13], reproduced with permission).

In Figure 4, the sector angle is denoted by $\Phi$, and the extent of the sector is given by radial distances $d_{\min }$ and $d_{\max }$. The image width is denoted by $w$. We also denote the total number of divisions along each radial line (axial resolution) by $m$, and the number of division of the sector angle (lateral resolution) by $n$. The Cartesian coordinates of the sampled points for radial-polar sampling are given by

$$
\begin{gathered}
d_{j}=d_{\min }+j\left(d_{\max }-d_{\min }\right) /(m-1) ; \theta_{i}=(3 \pi-\Phi) / 2+i \Phi /(n-1) \\
x(i, j)=d_{j} \cos \theta_{i}+w / 2 ; y(i, j)=-d_{j} \sin \theta_{i} ; i=0 \ldots(n-1) ; j=0 \ldots(m-1)
\end{gathered}
$$

The non-uniform spacing of points in the radial-polar sampling method causes the density of points to increase towards the sector's apex. The radial-uniform sampling method uses a constant arc length $\Delta$ between points along each arc to generate a uniform spacing between points. The equations for this sampling model are same as in Equation (1) except that the polar angle $\theta$ will now depend on both $i$ and $j$ as shown below.

$$
\theta_{i j}=(3 \pi-\Phi) / 2+i \Delta / d_{j}
$$

In the radial uniform sampling scheme, the parameter equivalent to the lateral resolution $n$ is the number of sampling points $n_{u}$ along an arc at distance $d_{i}$ given by

$$
n_{u}=\Phi d_{j} / \Delta
$$

The uniform grid is the simplest sampling model corresponding to a rectangular arrangement of uniformly spaced points with a constant distance $\delta$ between points. Even though the distribution of points does not correspond to a sectoral model, the uniform grid represents the scan geometry of a linear scan system, where the axial beams are parallel to each other. If a sector scan region is required, 
the points outside the region are clipped using the line equations of the two bounding edges. Using Equation (3), if $f\left(x, y, \theta_{\min }\right)>0$ or $f\left(x, y, \theta_{\max }\right)<0$, the point $(x, y)$ is outside the sector region.

$$
\begin{gathered}
\theta_{\min }=(3 \pi-\Phi) / 2 ; \theta_{\max }=(3 \pi+\Phi) / 2 \\
f(x, y, \theta)=(x-w / 2) \sin \theta+y \cos \theta
\end{gathered}
$$

More details and implementation aspects of the above three models are given in [14]. For speckle simulation, we use the method given in [7]. Their model is based on a complex distribution of incoherent phasors $(u, v)$ given by a two-dimensional Gaussian function $g_{\sigma}$. The complex amplitude of each pixel is initialized with the square-root of the sampled intensity value. The number of incoherent phasors $M(x, y)$ at each pixel $(x, y)$ is set as the value of a random number under a uniform distribution within a pre-specified range $[a, b]$. The incoherent phasors are generated and added $M$ times to both the real and imaginary components of the complex value at each pixel. The noisy intensity value is then given by the amplitude of the complex number.

After generating speckle noise at the sampled points, we use an interpolation method to fill the empty space left by the sampling step. In general, the interpolated value at a specified coordinate $(x, y)$ of an image $I$ is computed by grouping the sample values at neighboring pixels $(l, m)$ using the following formula [15]:

$$
I(x, y)=\sum_{l, m \in Z} \varphi(x-l, y-m) I(l, m)
$$

where, $\varphi()$ denotes a two-dimensional interpolation/synthesis function that provides the weights of the linear combination of sampled intensity values. Commonly used interpolation methods are B-Spline and cubic Hermite [16,17]. In [9], the authors used an interpolation scheme using the Lanczos-3 kernel [15,18-21].

\section{Synthetic Ultrasound Images}

The framework detailed above provides several options and parametric variations in each stage of the pipeline. As seen in Section 3, the three sampling methods and three interpolation schemes themselves give nine possible combinations. Each sampling scheme has its own set of parameters that can be varied over a wide range of values. The speckle noise generation algorithm also has a set of statistical parameters governing the noise distribution.

Figure 5 shows the variations when the axial resolution $m$ is increased in radial-polar sampling, keeping the lateral resolution fixed at $n=40$. The interpolation used was Lanczos-3 [19]. As the number of sampling points along axial beams is increased, we observe that the sector image becomes smoother and less grainy. When the value of $m$ is kept increasing with a fixed value for $n$, the overall clarity of features diminish because of close proximity of sampling points. Therefore, we need to carefully select the values of $m$ and $n$ to obtain the quality of real ultrasound images.

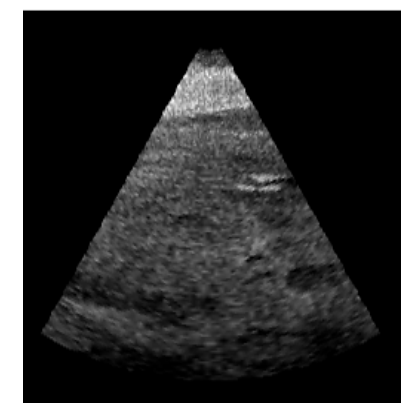

$m=120$

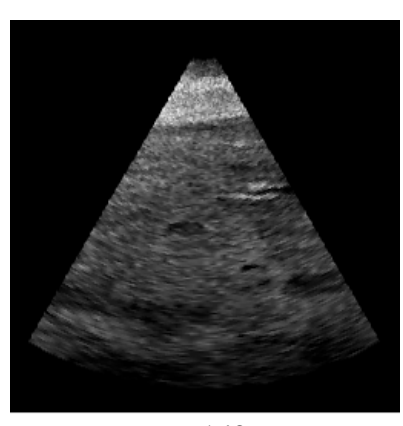

$m=160$

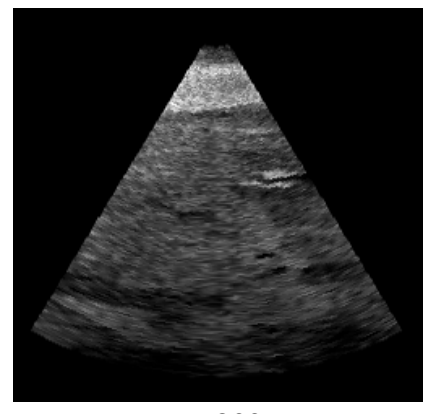

$m=200$

Figure 5. Effect of changing axial resolution $(m)$ in radial-polar sampling ([13], reproduced with permission). 
Similar results for radial uniform sampling are shown in Figure 6.

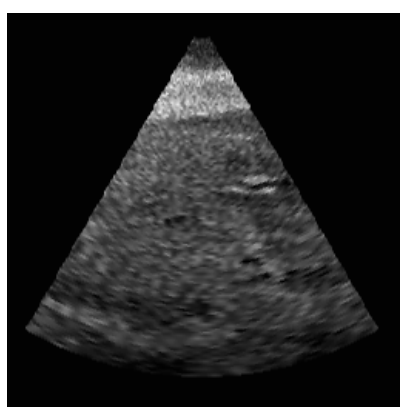

$m=120$

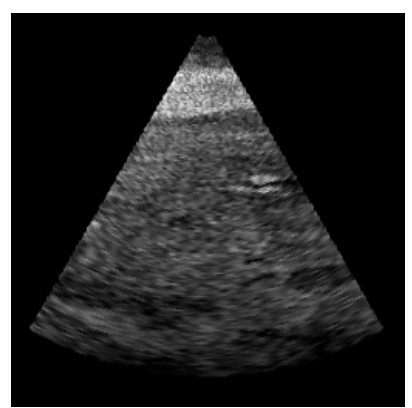

$m=160$

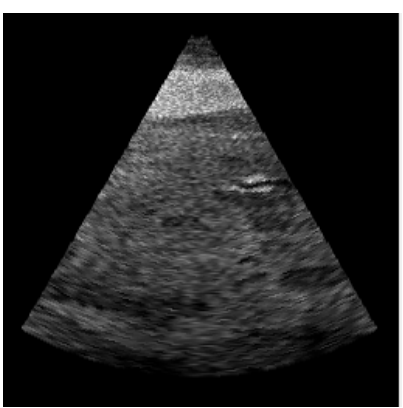

$m=200$

Figure 6. Effect of changing axial resolution $(m)$ in radial-uniform sampling ([13], reproduced with permission).

Some of the commonly found artifacts in simulated images when values of certain parameters become large are shown in Figure 7. In Figure 7a, a large value for $m$ results in a dense, overlapping set of points along beam directions resulting in smoothing/merging of pixels. A similar effect is seen when both $n$ and $m$ are large (Figure $7 \mathrm{~b}$ ). When the $\sigma$ value is large in the speckle generation function, the image becomes too grainy with loss of fine details, as in Figure 7c.

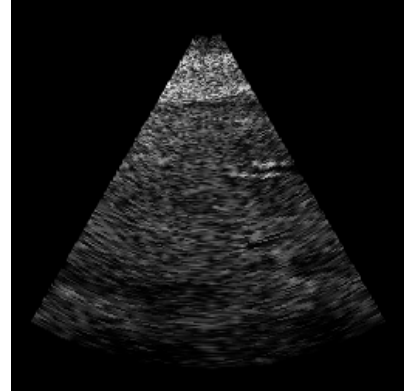

(a) $n=40, m=240, \sigma=1.7$

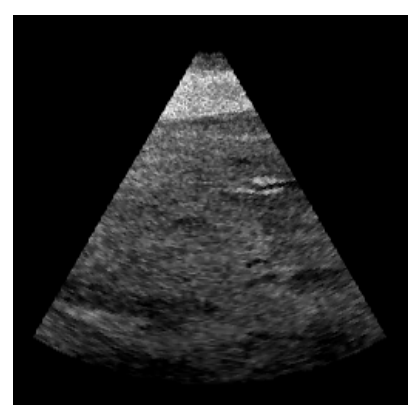

(b) $n=60, m=160, \sigma=0.5$

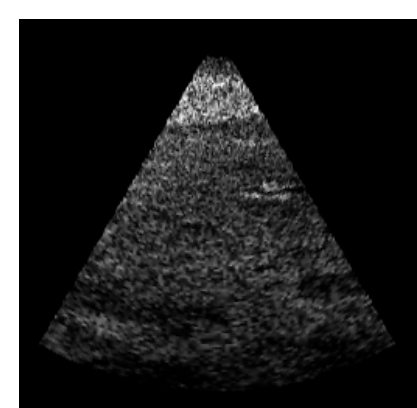

(c) $n=80, m=120, \sigma=1.9$

Figure 7. Image artifacts produced by large values of sampling and noise parameters ([13], reproduced with permission).

\section{Analysis of Texture Features}

One of the key requirements in the analysis of image modelling and simulation algorithms that use synthetic data is image quality assessment. Quality measures such as spatial frequency measure (SFM) and spectral activity measures (SAM) are commonly used in the assessment of image quality in the evaluation of compression and noise filtering algorithms [18]. In our prior work [13], we used entropy, SFM and SAM to compare the quality of the synthetic images with that of real ultrasound images. From that analysis, it became evident that we need to use higher order texture features to accurately characterize the desirable image properties, and to arrive at a robust set of quality metrics that conform to the subjective evaluations performed by expert clinicians. This paper presents a quality evaluation model using local binary patterns (LBP) and proposes a set of quality metrics (LBP feature values) that can provide consistent results on both synthetic and real ultrasound images. It has also been shown that synthetic images that give minimum feature deviation from real images are the ones that were given the highest subjective evaluation scores by experts, and therefore the proposed feature set contain sufficient information about the required texture features in the input synthetic images. In other words, these quality metrics determine the optimal set of sampling and noise parameters that would produce simulated ultrasound images closely matching the texture features of real ultrasound 
images. The images generated in this manner could be used to evaluate algorithms for speckle noise filtering (Figure 8).

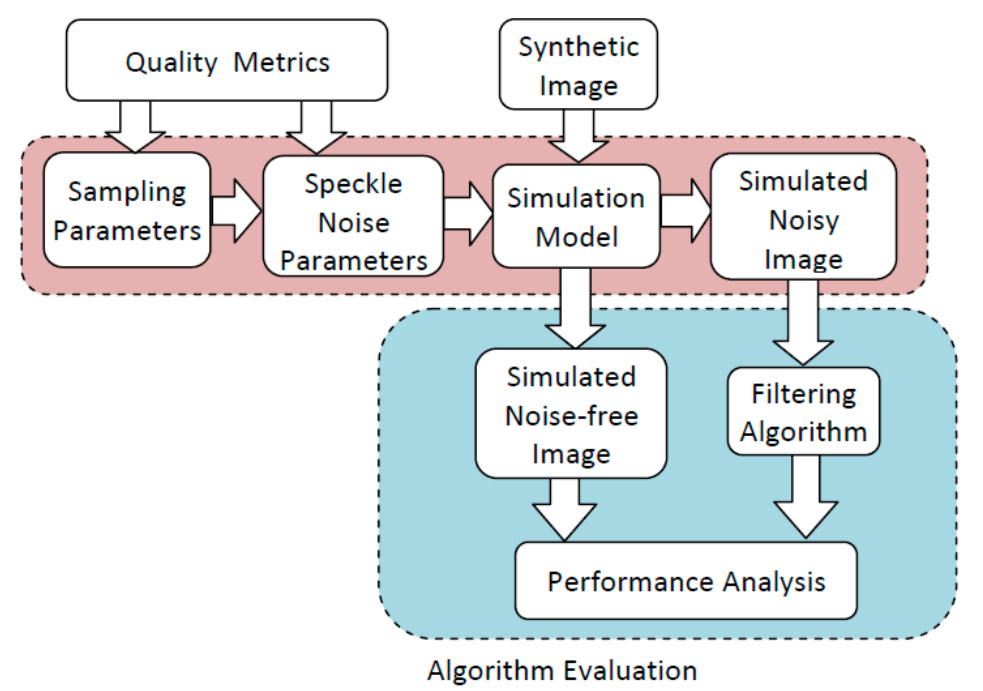

Figure 8. Application of the proposed local binary patterns (LBP) features in the evaluation of filtering algorithms.

\subsection{Local Binary Patterns (LBP)}

The irregularity and heterogeneity of texture features form the primary characteristics of ultrasound images and therefore play an important role in the assessment of their quality. A powerful texture descriptor that has been successfully applied in the field of medical image analysis is called the Local Binary Pattern (LBP) [22,23]. This feature is derived by comparing the intensity at each pixel with its eight neighbors and encoding the information in an 8-bit integer value. This encoding can be viewed as a transformation of the input image into an LBP image as shown in Figure 9.

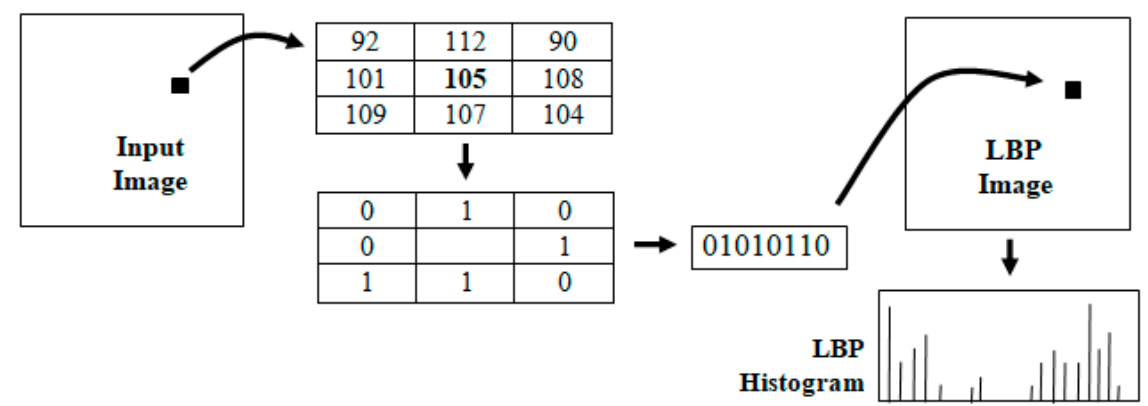

Figure 9. The intermediate steps in the computation of the LBP histogram of an image.

The histogram of the LBP image is generally used for texture classification [11]. In the area of medical image analysis, LBP methods have been successfully used in characterizing disease patterns [23]. There has also been a limited number of applications of local binary patterns in image quality assessment [10]. In this paper, we propose a novel approach for objective quality evaluation of synthetic ultrasound images using features derived from the LBP histogram. It is not necessary to use the entire range of the LBP histogram to characterize the texture attributes. The LBP histogram contains a few predominantly large and separable values that could be used as the primary features for texture analysis. 


\subsection{LBP Features of Synthetic Ultrasound Images}

The LBP histogram of ultrasound images contains predominant features that represent texture characteristics in the image. As an example, a synthetic ultrasound image of size $256 \times 256$ pixels, the corresponding LBP image and the LBP histogram are shown in Figure 10.

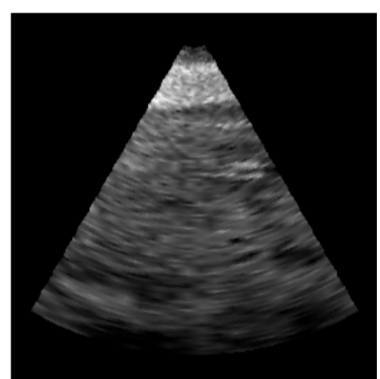

(a)

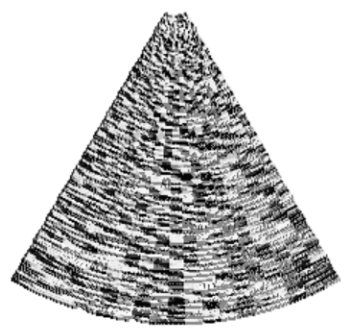

(b)

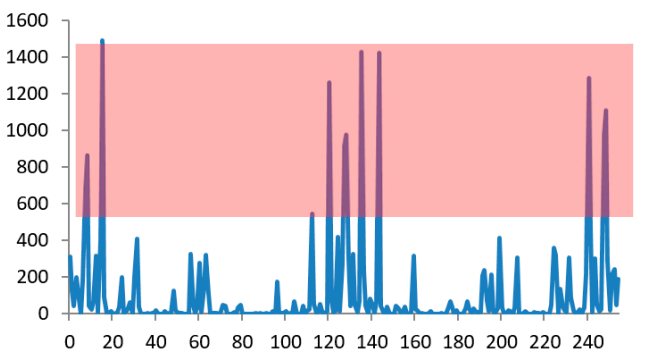

(c)

Figure 10. (a) A synthetic ultrasound image; (b) The LBP image; (c) the LBP histogram.

In Figure 10c, we have highlighted the important LBP features based on their magnitudes. The LBP histogram contains 256 values $L_{i}, i=0 \ldots 255$. We propose the following feature vector consisting of eight LBP features for quality assessment:

$$
V=\left\{L_{8}, L_{15}, L_{120}, L_{128}, L_{135}, L_{143}, L_{240}, L_{248}\right\}
$$

The above features show consistent variations with changes in axial and lateral resolutions at the modelling stage. The next section discusses the experimental results in detail and also shows that the LBP features can also help select these modelling parameters so that the features closely match with those of real ultrasound images.

\section{Experimental Analysis and Validation}

\subsection{LBP Feature Vector for Reference Images}

The feature vector given in Equation (6) was computed for the three reference images in Figure 1, and the average of the three sets were used as the reference feature vector. These values are given below:

$$
V_{\text {Ref }}=\{447.3,597.3,508.7,433.7,691.7,435.3,459.3,290\}
$$

The reference values were used in our experimental analysis detailed in the following sections, to find the answers to three main research questions:

1. When the parameters controlling the resolution in a sampling method are adjusted from coarse to fine, do the values of the corresponding LBP feature vector consistently tend towards the reference feature vector?

2. Do the synthetic images that give feature values close to the reference vector also have consistently high subjective evaluation scores assigned by clinical experts?

3. Which one of the three modelling schemes generated feature values that are closest to the reference feature vector?

We performed an extensive analysis using LBP features by varying the sampling parameters for the radial polar, radial uniform and uniform grid methods, and the results are summarized below. 


\subsection{LBP Feature Vector for Radial-Polar Sampling}

Here, we consider the images generated using the radial-polar sampling scheme. Quality analysis using global features such as entropy, SAM and SFM, and also subjective evaluations revealed that an axial resolution value ( $m$ in Equation (1)) of 120, and speckle noise level $\sigma=0.5$ gave acceptable results. We therefore fixed these parameters and varied only the lateral resolution ( $n$ in Equation (1)) from 10 to 120. A few sample images are shown in Figure 11.

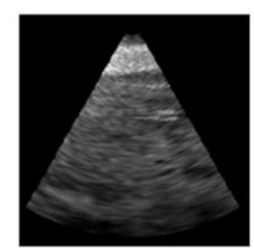

$n_{u}=20$

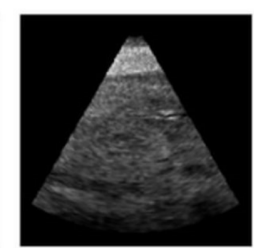

$n_{u}=40$

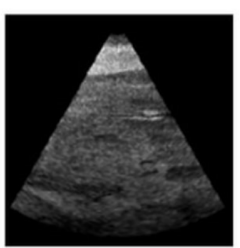

$n_{u}=60$

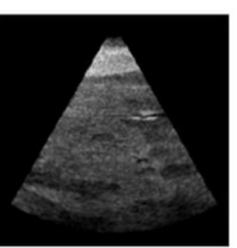

$n_{u}=80$

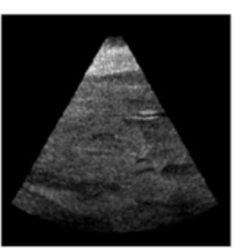

$n_{u}=100$

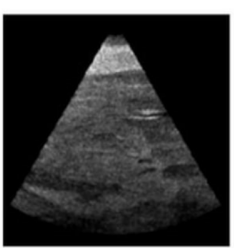

$n_{u}=120$

Figure 11. Synthetic images generated using radial polar sampling with a coarse to fine variation of lateral resolution parameter $n$.

The variations of each of the eight components of the feature vector (Equation (6)) computed from the LBP histogram are shown in Figure 12.
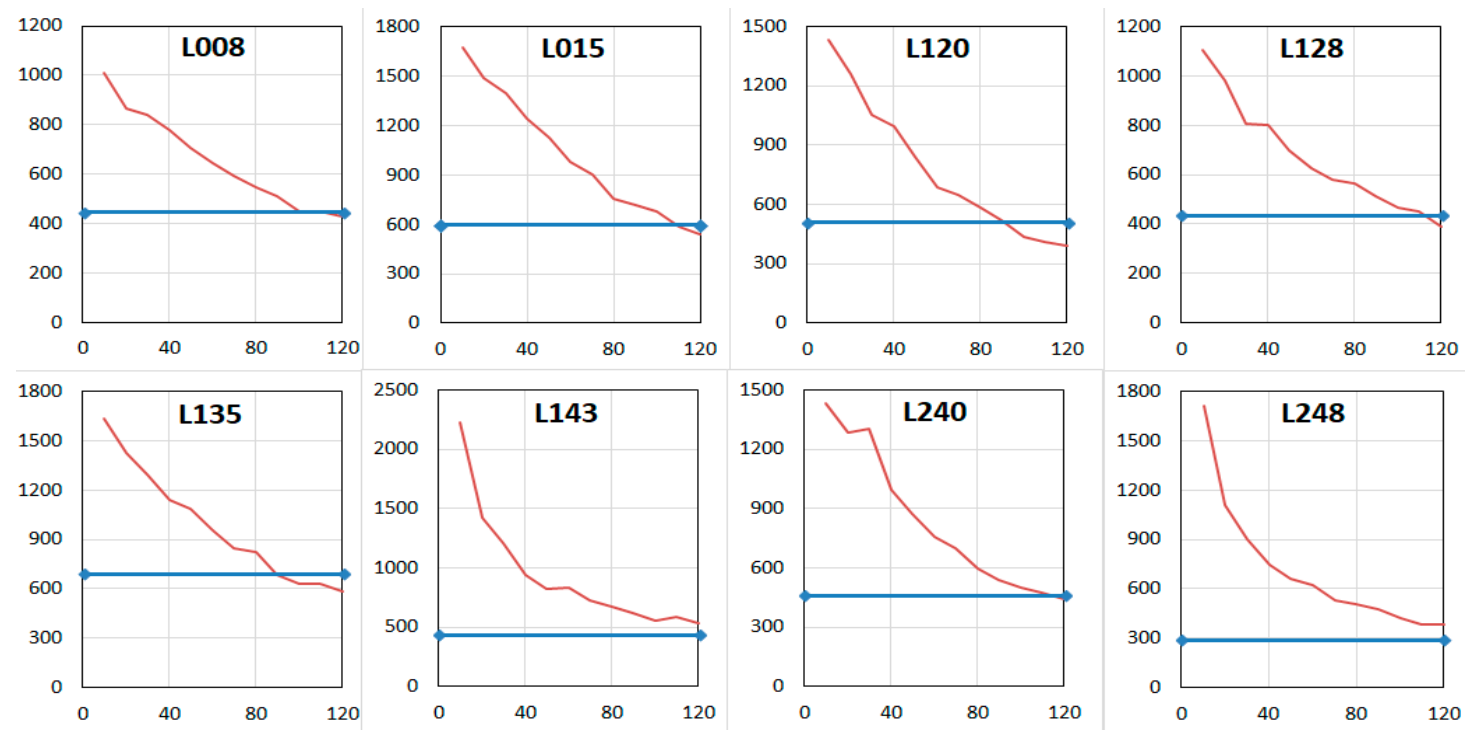

Figure 12. Variations of LBP feature vector components with lateral resolution in radial-polar sampling. The $x$-axis gives the values of $n$. The $y$-axis gives the range of values of an LBP feature shown in the chart title.

In Figure 12, the blue horizontal lines indicate the values of the reference feature vector as given in Equation (7). All components of the LBP feature vector show consistent variations towards the reference values as the value of the lateral resolution parameter $n$ is increased.

\subsection{LBP Feature Vector for Radial-Uniform Sampling}

The parameter affecting the lateral resolution in radial uniform sampling is $n_{u}$ given in Equation (3). The effect of variation of this parameter in the quality of the synthetic images is shown in Figure 13. 


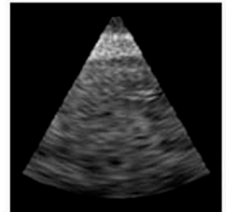

$n_{u}=20$

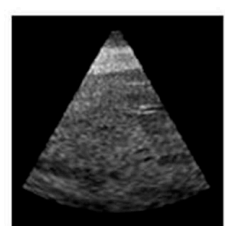

$n_{u}=40$

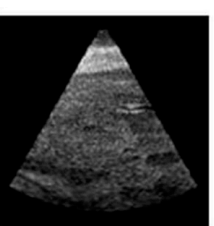

$n_{u}=60$

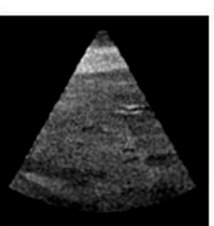

$n_{u}=80$

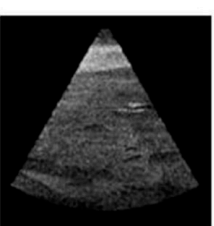

$n_{u}=100$

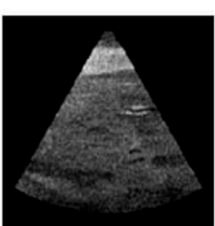

$n_{u}=120$

Figure 13. Synthetic images generated using radial uniform sampling with a coarse to fine variation of lateral resolution parameter $n_{u}$.

The variations of the LBP feature vectors (Equation (6)) with $n_{u}$ are shown in the graphs in Figure 14.
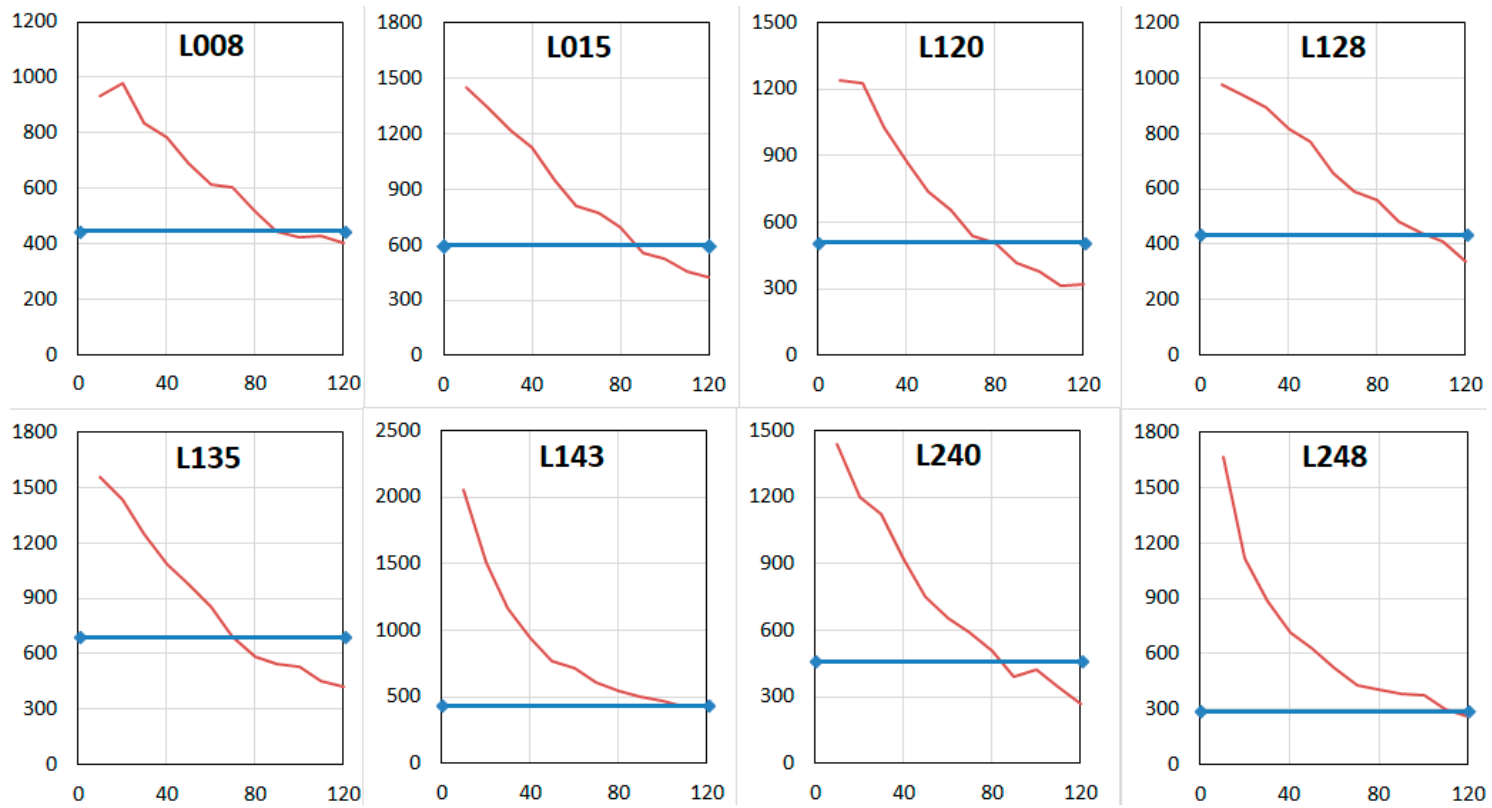

Figure 14. Variations of LBP feature vector components with lateral resolution in radial-uniform sampling. The $x$-axis gives the values of $n_{u}$. The $y$-axis gives the range of values of an LBP feature shown in the chart title.

In the case of radial uniform sampling also, we see a trend towards the reference values of the LBP features as the lateral resolution $n_{u}$ of the images is increased from 10 to 120 .

\subsection{LBP Feature Vector for Uniform-Grid Sampling}

As previously mentioned in Section 3, the uniform-grid sampling method uses a constant spacing $\delta$ between sampling points along both $x$ and $y$ directions. Therefore, increasing $\delta$ reduces the resolution of the sampled image in both directions. Consequently, we will get a fine to coarse variation of quality in the image as $\delta$ is increased, as shown in Figure 15.

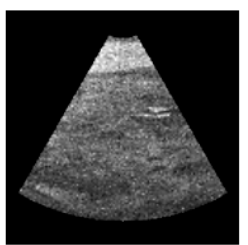

$\delta=2$

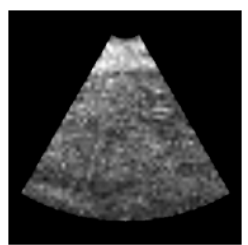

$\delta=4$

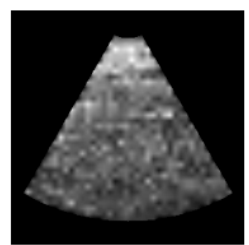

$\delta=6$

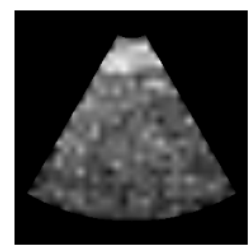

$\delta=8$

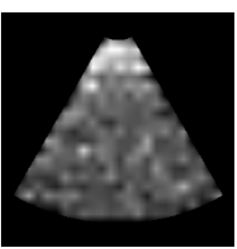

$\delta=10$

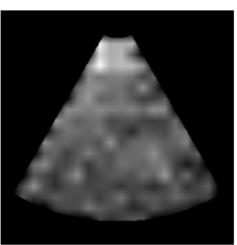

$\delta=12$

Figure 15. Synthetic images generated using uniform-grid sampling scheme with increasing values of the grid spacing parameter $\delta$. 
Since the image quality deteriorates as the value of $\delta$ is increased, the corresponding values of the LBP feature vector deviates further from the reference feature vector, as shown in Figure 16.
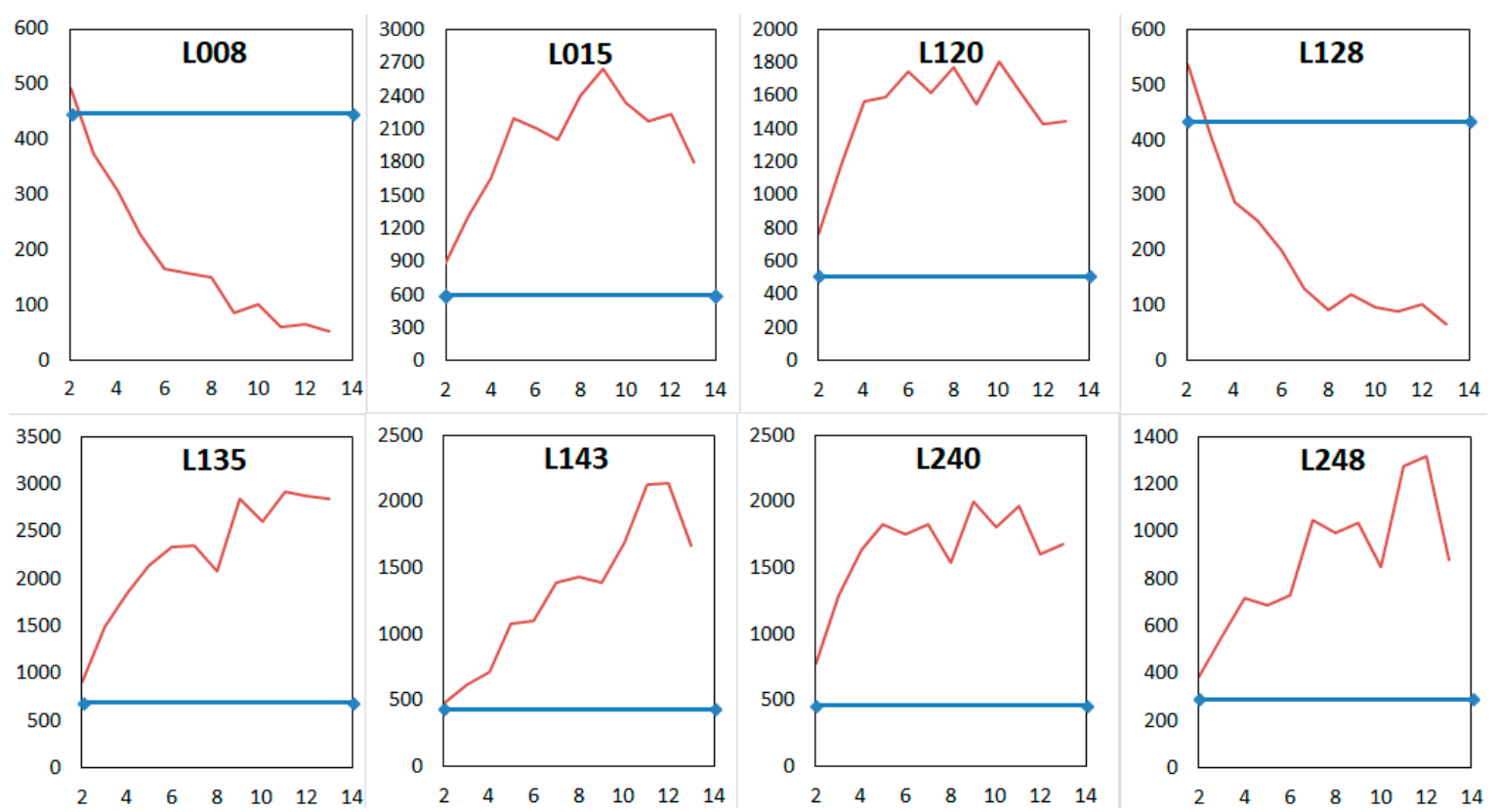

Figure 16. Variations of LBP feature vector components with grid spacing in uniform-grid sampling. The $x$-axis gives the values of $\delta$. The $y$-axis gives the range of values of an LBP feature shown in the chart title.

\subsection{Comparative Analysis of Sampling Techniques}

The objective quality of the synthetic images produced by the three sampling methods is evaluated by computing the closest distance of the LBP feature vectors from the reference feature vector (indicated by the blue horizontal lines in Figures 12, 14 and 16) and the measure features of synthetic images (red colored lines in Figures 12, 14 and 16) using a Euclidean distance metric. The plots of the distance values for the three methods are given in Figure 17, along with the location of the minimum distance in each case.

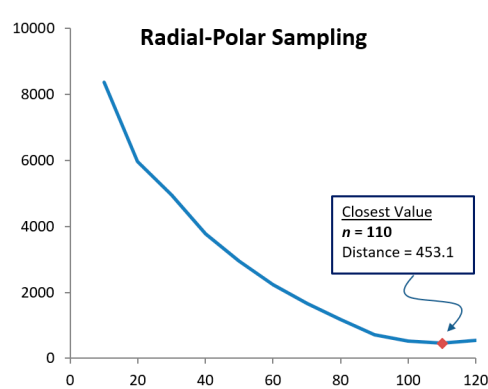

(a)

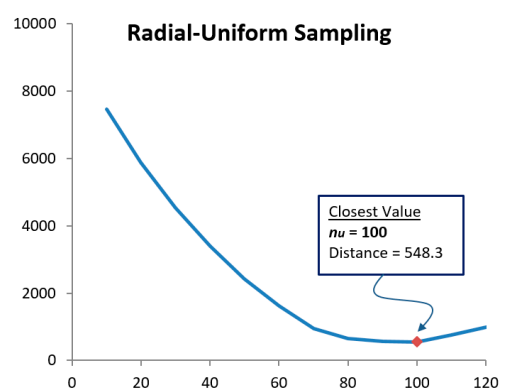

(b)

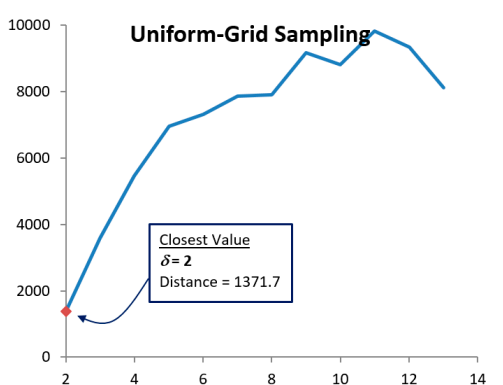

(c)

Figure 17. Plots showing the closest matching positions of the LBP feature vector with reference vector for images generated using (a) radial-polar sampling; (b) radial-uniform sampling; (c) uniform-grid sampling.

The comparative analysis showed that radial polar sampling with lateral resolution $n=110$ gave the closest distance of the LBP feature vector from the reference feature vector. This result also matches very closely with the subjective evaluation scores reported in [13]. 


\section{Conclusions and Future Work}

This paper has presented the complete framework for generating realistic and simulated ultrasound images incorporating image acquisition models, speckle noise formation processes and image interpolation schemes. The paper has introduced three sampling schemes, viz., radial-polar, radial-uniform and uniform grid sampling methods. These methods together with the speckle simulation model and the interpolation scheme formed the simulation model of the processing pipeline. These processes within the simulation model allows users to vary a wide range of parameters that control the image and noise formation processes. The simulated images with speckle noise could be used to evaluate noise filtering methods as ground truth data (the corresponding synthetic images without noise) are readily available.

The main contributions of the paper include a method for objective quality assessment of synthetic images using texture features derived from local binary patterns and detailed experimental analysis, that showed the effectiveness of LBP features in representing changes in image quality with modeling parameters. More importantly, the values of the LBP features approached the values computed from real synthetic images in a systematic way for the following parametric variations:

- Radial polar: when the parameter $n$ is increased from 10 to 110

- Radial uniform: when the parameter $n_{u}$ is increased from 10 to 100

- Uniform grid: when the spacing parameter $\delta$ is reduced from 14 to 2

The above analysis provided us an optimal set of modeling parameters that could be used for generating realistic synthetic images. The study also revealed that synthetic images generated by radial polar sampling technique gave better quality outputs compared to other sampling methods. The quality of such synthetic images was further validated using subjective evaluations performed by clinical experts.

Future work is directed towards using the proposed algorithm on a wider range of ultrasound images to analyse deviations in the values of LBP feature components and to evaluate the robustness of the selected LBP features under variations in imaged objects and tissue characteristics. The distribution of speckle noise also is dependent on the imaged tissue characteristics and therefore not uniform across the image. Variations in speckle distribution could be modelled by overlaying on the sampled points regions with assigned weights to control the influence of noise within the selected regions.

The method proposed and the quality analysis presented in this paper could be used for generating a synthetic ultrasound image dataset. This dataset would find applications in the performance evaluation of filtering systems.

Acknowledgments: The permission granted by Joe Antony, MD, for using three images from the Ultrasound Image Gallery [7] as reference images in our work (Figure 1) is gratefully acknowledged.

Author Contributions: P. Singh and R. Mukundan designed and implemented the algorithms and carried out experimental analysis as described in the paper. R. De Ryke provided guidance on important texture features required in ultrasound images and also provided subjective evaluations of the generated synthetic images.

Conflicts of Interest: The authors declare no conflict of interest.

\section{References}

1. Zhang, J.; Cui, W.; Wu, L.; Lin, G.; Cheng, Y. A novel algorithm based on wavelet-trilateral filter for de-noising medical ultrasound images. In Proceedings of the Control and Decision Conference 2016, Yinchuan, China, 28-30 May 2016; pp. 3804-3809. [CrossRef]

2. Malutan, R.; Terebes, R.; Germain, C.; Borda, M.; Cislariu, M. Speckle noise removal in ultrasound images using sparse code shrinkage. In Proceedings of the IEEE International Conference on E-Health and Bioengineering 2015, Iasi, Romania, 19-21 November 2015; pp. 1-4. [CrossRef]

3. Le, T. Adaptive noise reduction in ultrasound imaging. In Proceedings of the IEEE Symposium on Signal Processing in Medicine and Biology, Philadelphia, PA, USA, 13 December 2014; p. 1. [CrossRef]

4. Zang, X.; Bascom, R.; Gilbert, C.; Toth, J.; Higgins, W. Methods for 2D and 3D endobronchial ultrasound image segmentation. IEEE Trans. Biomed. Eng. 2016, 63, 1426-1439. [CrossRef] [PubMed] 
5. Cortes, C.; Kabongo, K.; Macia, I.; Ruiz, O.E.; Florez, J. Ultrasound image dataset for image analysis algorithms evaluation. In Innovation in Medicine and Healthcare. Smart Innovation, Systems and Technologies; Chen, Y.W., Torro, C., Tanaka, S., Howlett, R., Jain, L., Eds.; Springer: Cham, Germany, 2016; Volume 45, pp. 447-457. [CrossRef]

6. Telmed Ultrasound Medical Systems. Available online: http://www.pcultrasound.com/products/products_ usimg/index.html (accessed on 30 January 2017).

7. Antony, J. Ultrasound Image Gallery. Available online: http://www.ultrasound-images.com/ (accessed on 15 March 2017).

8. Loizou, C.P.; Pattichis, C.S. Despeckle Filtering for Ultrasound Imaging and Video; Algorithms and Software; Morgan \& Claypool: San Rafael, CA, USA, 2015; Volume I, p. 180. ISBN 978-1-62705-668-7.

9. Perreault, C.; Auclair-Fortier, M.F. Speckle simulation based on B-mode echographic image acquisition model. In Proceedings of the 4th Canadian Conference on Computer and Robot Vision (CRV 2007), Montreal, QC, Canada, 28-30 May 2007; pp. 379-386. [CrossRef]

10. Singh, P.; Mukundan, R.; de Ryke, R. Quality analysis of synthetic ultrasound images using co-occurrence texture statistics. In Proceedings of the Image and Vision Computing (IVCNZ2017), Christchurch, New Zealand, 4-6 December 2017.

11. Pietikainen, M.; Zhao, G.; Hadid, A.; Ahonen, T. Computer Vision Using Local Binary Patterns; Springer: London, UK; pp. E1-E2. ISBN 978-0-85729-748-8.

12. Zhang, M.; Xie, J.; Zhou, X.; Fujita, H. No reference image quality assessment based on local binary pattern statistics. In Proceedings of the Visual Communications and Image Processing (VCIP) 2013, Kuching, Malaysia, 17-20 November 2013. [CrossRef]

13. Singh, P.; Mukundan, R.; de Ryke, R. Modelling, Speckle Simulation and Quality Evaluation of Synthetic Ultrasound Images. In Communications in Computer and Information Science; Hernández, M., Gonzalez-Castro, V., Eds.; Springer International Publishing: Cham, Germany, 2017; pp. 74-85. [CrossRef]

14. Singh, P.; Mukundan, R.; de Ryke, R. Synthetic Models of Ultrasound Image Formation for Speckle Noise Simulation and Analysis. In Proceedings of the IEEE International Conference on Signals and Systems 2017, Bali, Indonesia, 16-18 May 2017; pp. 278-284. [CrossRef]

15. Somawirata, I.K.; Uchimura, K.; Koutaki, G. Image enlargement using adaptive manipulation interpolation kernel based on local image data. In Proceedings of the IEEE International Conference on Signal Processing, Communication and Computing 2012, Hong Kong, China, 12-15 August 2012; pp. 474-478. [CrossRef]

16. Li, K.-Y.; Wang, W.-D.; Zheng, K.-W.; Liu, W.-B.; Xu, G.-L. The application of B-spline based interpolation in real-time image enlarging processing. In Proceedings of the 2nd International Conference on Systems and Informatics 2014, Shanghai, China, 15-17 November 2014; pp. 823-827. [CrossRef]

17. Goceri, E.; Lomenie, N. Interpolation approaches and spline based resampling for MR images. In Proceedings of the 5th International Symposium on Health Informatics and Bioinformatics, Antalya, Turkey, 20-22 April 2010; pp. 137-143. [CrossRef]

18. Xia, Z.W.; Li, Q.; Wang, Q. Quality metrics of simulated intensity images of coherent ladar. In Proceedings of the International Conference on Optoelectronics and Microelectronics, Jilin, China, 23-25 August 2012; pp. 214-216. [CrossRef]

19. Mirza, S.; Kumar, R.; Shakher, C. Study of various preprocessing schemes and wavelet filters for speckle noise reduction in digital speckle pattern interferometric fringes. Opt. Eng. 2005, 44. [CrossRef]

20. Grgic, S.; Grgic, M.; Mrak, M. Reliability of objective picture quality measures. J. Electr. Eng. 2004, 55, 3-10.

21. Burger, W.; Burge, M.J. Digital Image Processing: An Algorithmic Introduction Using Java; Springer: London, UK, 2008; p. 565. ISBN 978-1-84628-968-2.

22. Zhang, J.; Marszalek, M. Local features and kernels for classification of texture and object categories: A comparative study. Int. J. Comput. Vis. 2007, 73, 213-238. [CrossRef]

23. Sørensen, L.; Shaker, S.B.; de Bruijne, M. Quantitative Analysis of Pulmonary Emphysema using Local Binary Patterns. IEEE Trans. Med. Imaging 2010, 29, 559-569. [CrossRef] [PubMed]

(C) 2017 by the authors. Licensee MDPI, Basel, Switzerland. This article is an open access article distributed under the terms and conditions of the Creative Commons Attribution (CC BY) license (http:// creativecommons.org/licenses/by/4.0/). 